\title{
AN OPTIMUM DESIGN PROBLEM IN MAGNETOSTATICS *
}

\author{
Antoine Henrot ${ }^{1}$ And GrÉGory Villemin ${ }^{2}$
}

\begin{abstract}
In this paper, we are interested in finding the optimal shape of a magnet. The criterion to maximize is the jump of the electromagnetic field between two different configurations. We prove existence of an optimal shape into a natural class of domains. We introduce a quasi-Newton type algorithm which moves the boundary. This method is very efficient to improve an initial shape. We give some numerical results.
\end{abstract}

Mathematics Subject Classification. 49J20, 49Q10, 65K10, 78A30.

Received: April 17, 2001. Revised: October 11, 2001.

\section{INTRODUCTION}

Let us consider a magnet $\Omega_{0}$ which is located near a toothed wheel $K$. The magnetic field created by $\Omega_{0}$ is of course different when it is a tooth of $K$ which is faced to the magnet or when it is a hollow of the wheel. The first situation will be denoted in the following by the superscript $T$ and the second one, by $H$. For example, $\mathbf{B}^{T}$ will be the magnetic vector induction when $\Omega_{0}$ is faced to a tooth and $\mathbf{B}^{H}$ when it is faced to a hollow, see Figure 1. This kind of system is present in several technological devices (engine of a car, for example) and it is exactly the difference of the magnetic signals between the two situations which releases the desired action. In order to be very precise, the jump in the signal has to be the larger possible (with respect to some technological constraints). Therefore, an idea is to choose a shape of the magnet which allows to reach such a goal. The aim of this paper is to do a mathematical and numerical study of this problem.

In the sequel of this Introduction, we fix the notations, the constraints and we present a modelling of the problem as a tri-dimensional shape optimization (or optimum design) problem. In Section 2, we prove the existence of an optimum magnet in the natural class of uniformly Lipschitz open sets. In Section 3, we present the discretization and the numerical method we have followed to approximate an optimal domain. At last, Section 4 is devoted to numerical results and examples.

Let us first establish the set of equations which give the magnetic induction $\mathbf{B}$ for a given configuration. We will denote by $\Omega_{0}$ the place occupied by the magnet and $\Gamma_{0}$ its boundary, $K$ the toothed wheel, $\Omega_{1}$ is the air surrounding the magnet and the wheel. We also introduce $\Omega=\overline{\Omega_{0}} \cup \Omega_{1}=\mathbb{R}^{3} \backslash K$ and denote by $\Gamma=\partial \Omega=\partial K$

\footnotetext{
Keywords and phrases. Shape optimization, optimum design, magnet, numerical examples.

* This work has been done thanks to a Grant of a firm from Besançon (France): Moving Magnet Technologies that we want to thank here.

${ }^{1}$ École des Mines de Nancy and Institut Elie Cartan, BP 23954506 Vandœuvre-lès-Nancy, France.

e-mail: henrot@iecn.u-nancy.fr

2 Centre de Recherche Valeo - Systèmes d'essuyage, Z.A. de l'Agiot, 78231 La Verrière, France.

e-mail: gregory.villemin@valeo.com
} 

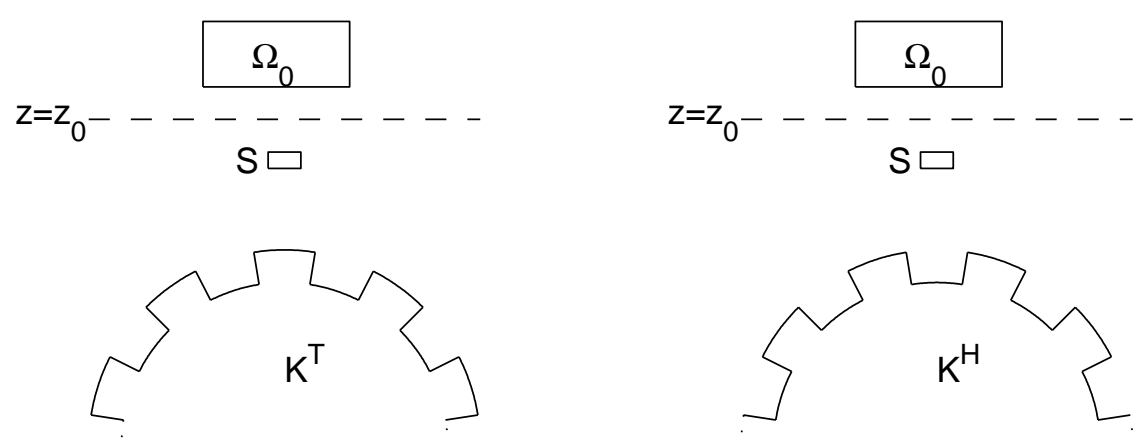

Figure 1. Magnet faced to a tooth (left), faced to a hollow (right).

its boundary. In the previous notations, we consider the sets $\Omega, \Omega_{0}, \Omega_{1}$ to be open connected domains in $\mathbb{R}^{3}$ and we assume their boundaries to be enough regular (at least Lipschitz). At last, we assume that the sound $S$ is a compact regular set.

For all the quantities described below and the classical equations of magnetostatic, we refer e.g. to [5] or [7]. We will denote by $\mathbf{B}$ the magnetic induction vector, $\mathbf{H}$ the magnetic vector field and $\mathbf{M}$ the magnetization vector linked to the two previous quantities by the relation

$$
\mathbf{B}=\mu_{0}(\mathbf{H}+\mathbf{M})
$$

(with $\mu_{0}=4 \pi 10^{-7}$ ). Since we are in a magnetostatic context, Maxwell's equations can be written here in each medium separately as

$$
\left\{\begin{array}{l}
\operatorname{curl} \mathbf{H}=0 \\
\operatorname{div} \mathbf{B}=0 .
\end{array}\right.
$$

Due to the first equation in (2), we are led to work with the scalar magnetic potential $\varphi$ defined (up to a constant) such that $\mathbf{H}=-\nabla \varphi$.

Let us describe more precisely the equations in each media. We assume the magnet to be perfect and we choose its orientation in such a way that its magnetization is vertical. Therefore, if $B_{r}$ is the retentivity of the magnet, we will set $M_{0}=\frac{B_{r}}{\mu_{0}}$ and $\mathbf{M}_{\mathbf{0}}=M_{0} \vec{k}$. This yields that $\mathbf{M}$ is given in each media by

$$
\mathbf{M}=\left(\mu_{r}-1\right) \mathbf{H}+\mathbf{M}_{\mathbf{0}}
$$

where $\mu_{r}$ is the relative permeability of the media. Now,

- in the air $\mu_{r}=1$ and $M_{0}=0$, so (1) and (3) yield $\mathbf{B}=\mu_{0} \mathbf{H}=-\mu_{0} \nabla \varphi$,

- in the magnet $\mu_{r}=1$ and $M_{0} \neq 0$, so (1) and (3) yield $\mathbf{B}=\mu_{0}\left(\mathbf{H}+\mathbf{M}_{\mathbf{0}}\right)=\mu_{0}\left(\mathbf{M}_{\mathbf{0}}-\nabla \varphi\right)$.

We express now the second equation in (2). In the air $\operatorname{div} \mathbf{B}=-\mu_{0} \Delta \varphi=0$, while inside the magnet div $\mathbf{B}=$ $-\mu_{0} \Delta \varphi+\mu_{0} \operatorname{div} \mathbf{M}_{\mathbf{0}}=0$. Now, since $\mathbf{M}_{\mathbf{0}}=M_{0} \vec{k}$ is constant its divergence vanishes and we still obtain $-\mu_{0} \Delta \varphi=0$ in the magnet. In conclusion $\Delta \varphi=0$ in $\Omega_{0} \cup \Omega_{1}$.

It remains to express the magnetic induction field at the interface between the magnet and the air and to find the boundary conditions. The classical condition at the interface is the continuity of the magnetic flux (continuity of the normal component of $\mathbf{B}$ ). According to the expressions of $\mathbf{B}$, this condition gives

$$
-\mu_{0} \nabla \varphi_{\Omega_{1}} \cdot \mathbf{n}=-\mu_{0} \nabla \varphi_{\Omega_{0}} \cdot \mathbf{n}+\mu_{0} \mathbf{M}_{\mathbf{0}} \cdot \mathbf{n}
$$


where the subscript denotes the media where the potential $\varphi$ has to be considered and $\mathbf{n}$ is the exterior normal vector to $\Gamma_{0}$. In other terms, the jump of the normal derivative of $\varphi$ across the boundary of the magnet is equal to $\mathbf{M}_{\mathbf{0}} . \mathbf{n}$. It means that, if we consider $\varphi$ to be globally defined in the whole domain $\Omega$, its Laplacian is a measure supported by $\Gamma_{0}$ of intensity $\mathbf{M}_{\mathbf{0}} . \mathbf{n}$ :

$$
-\Delta \varphi=L_{0} \quad \text { in } \Omega
$$

where $L_{0}$ is the linear form defined on the weighted Sobolev space $W_{0}^{1}(\Omega)$ (for a precise definition of this space which corresponds to the classical Sobolev space $H_{0}^{1}$ with the weight $\left(1+r^{2}\right)^{-1 / 2}$, we refer to [5] XI B, Def. 2) by

$$
\left\langle L_{0}, w\right\rangle:=\int_{\Gamma_{0}} \mathbf{M}_{\mathbf{0}} \cdot \mathbf{n} w \mathrm{~d} \sigma
$$

This linear form is well defined and continuous on $W_{0}^{1}(\Omega)$ by continuity of the trace operator. Let us now establish the boundary conditions: since the wheel is an equipotential, and according to the fact that $\varphi$ is defined up to a constant, we can fix $\varphi=0$ on $\partial \Omega$. At last, since there is no current at infinity, we have $\varphi(X) \rightarrow 0$ when $|X| \rightarrow+\infty$.

To sum up, we have obtained the following set of equations which define $\varphi$ in an unique way

$$
\left\{\begin{aligned}
-\Delta \varphi & =L_{0} & & \text { in } \Omega \\
\varphi & =0 & & \text { on } \partial \Omega \\
\varphi(X) & \rightarrow & & \text { when }|X| \rightarrow+\infty .
\end{aligned}\right.
$$

The problem (7) is well posed (see e.g. [5]). Introducing the fundamental solution of $-\Delta u_{\infty}=L_{0}$ in $\mathbb{R}^{3}$ defined by

$$
u_{\infty}(X)=\frac{1}{4 \pi} \int_{\Gamma_{0}} \frac{\mathbf{M}_{\mathbf{0}} \cdot \mathbf{n}}{|X-Y|} \mathrm{d} \sigma_{Y}
$$

we can decompose $\varphi$ as

$$
\varphi=u_{\infty}-v
$$

where $v$ is the harmonic function, taking the value $u_{\infty}$ on $\Gamma$ and vanishing at infinity. In other words, we can choose an integral formulation to solve (7). It consists in writing $\varphi$ as (simple layer potential)

$$
\varphi(X)=\frac{1}{4 \pi} \int_{\Gamma_{0}} \frac{\mathbf{M}_{\mathbf{0}} \cdot \mathbf{n}}{|X-Y|} \mathrm{d} \sigma_{Y}-\frac{1}{4 \pi} \int_{\Gamma} \frac{\alpha(Z)}{|X-Z|} \mathrm{d} \sigma_{Z}
$$

where the function $\alpha$ is obtained by solving the 1st kind Fredholm integral equation (expressing the boundary condition on $\Gamma$ ):

$$
\forall X \in \Gamma: \quad \int_{\Gamma_{0}} \frac{\mathbf{M}_{\mathbf{0}} \cdot \mathbf{n}}{|X-Y|} \mathrm{d} \sigma_{Y}=\int_{\Gamma} \frac{\alpha(Z)}{|X-Z|} \mathrm{d} \sigma_{Z}
$$

We must now describe the criterion we want to optimize. Physically, the magnetic field is measured by a sound $S$ located between the magnet and the wheel, see Figure 1. We recall that we have to consider two different situations. If $\omega$ is a given magnet, we will denote by $\varphi_{\omega}^{T}$ (or simply $\varphi^{T}$ if there is no misunderstanding) the magnetic potential when $\omega$ is faced to a tooth and $\varphi_{\omega}^{H}$ (or simply $\varphi^{H}$ ) when it is faced to a hollow. Since we 
want to find some magnet $\omega$ such that the gap between the two situations is maximum, we choose to introduce the following functional:

$$
J(\omega)=\frac{1}{2} \int_{S}\left|\nabla \varphi_{\omega}^{T}(X)\right|^{2}-\left|\nabla \varphi_{\omega}^{H}(X)\right|^{2} \mathrm{~d} X
$$

Of course, we need to put some constraints on the class of admissible magnets. For obvious technical reasons, we fix a maximum value $V_{0}$ for the volume. In the paper, we will denote by $|\omega|$ the volume of the set $\omega$ and by $|\partial \omega|$ its surface area. Moreover, in order to avoid that the magnet comes in contact with the sound or the wheel, we will fix a lower plane $z=z_{0}$ above which the magnet must hold, see Figure 1 . In the numerical approach, the volume constraint will be treated by penalization: we will substract to the functional $J$ a term like $r\left[\left(|\omega|-V_{0}\right)^{+}\right]^{2}$ (where $(A)^{+}$denotes, as usual, the positive part of the number $A$ ) with a large parameter $r$ of penalization. Therefore the functional we really maximize is

$$
J_{r}(\omega):=\frac{1}{2} \int_{S}\left|\nabla \varphi_{\omega}^{T}(X)\right|^{2}-\left|\nabla \varphi_{\omega}^{H}(X)\right|^{2} \mathrm{~d} X-r\left[\left(|\omega|-V_{0}\right)^{+}\right]^{2} .
$$

The other constraint will be treated by projection.

The aim of this work is to look for a domain $\Omega$ satisfying the constraints and which maximizes the functional $J$ given in (12).

\section{An EXistence Result}

\subsection{Introduction}

In this section, we will prove existence of a maximizer for the functional $J$ in the natural class of uniformly Lipschitz open sets in $\mathbb{R}^{3}$. This class can also be described as sets with the $\varepsilon$-cone property, and it has been introduced by Chenais in her important work, see $[3,4]$. In Section 1.2 , we recall the definition of the $\varepsilon$-cone property. We prove, in particular, that sets in this class with bounded volume have also an uniformly bounded area. In Section 1.3, we prove that the functional $J$ is estimated from above in this class. In the sequel, our approach is a classical one: we work with a maximizing sequence and Hausdorff convergence. The difficulty in our case is that the sets we consider are a priori unbounded and could have components which go to infinity. Now, if we want to use the compactness property of the Hausdorff convergence, we need to work with domains which are all contained in some fixed ball. That is why we will distinguish, in the proof of existence, components of the maximizing sequence which go to infinity of those which stay at finite distance of the wheel (see Sect. 1.5).

Here the result of continuity with respect to the domain that we need is not too difficult to get, because domain variation is only expressed in the linear form in the right hand side of the equation. Moreover, since the functional $J$ has to be calculated on a region $S$ where all the functions are harmonic, we can use the powerful properties of convergence of sequence of harmonic functions.

\subsection{The class of admissible sets}

We recall here the definition of the $\varepsilon$-cone property (see $[1,3,4,8]$ ).

Definition 1.1. Let $Y$ a point in $\mathbb{R}^{3}, \xi$ a unit vector and $\varepsilon$ a positive number, $\varepsilon<\pi / 2$. We introduce the cone $C(Y, \xi, \varepsilon)$ as the set defined by:

$$
C(Y, \xi, \varepsilon)=\left\{Z \in \mathbb{R}^{3},(Z-Y, \xi) \geq|Z-Y| \cos (\varepsilon) \text { and } 0<|Z-Y|<\varepsilon\right\}
$$


(where (.,.) denotes the usual scalar product in $\mathbb{R}^{3}$ and |.| the associated Euclidean norm).

We will say that an open set $\omega$ has the $\varepsilon$-cone property (with $\varepsilon>0$ is fixed), if

$$
\forall X \in \partial \omega, \exists \xi_{X} \text { a unit vector such that } \forall Y \in \bar{\omega} \cap B(X, \varepsilon) C\left(Y, \xi_{X}, \varepsilon\right) \subset \omega .
$$

Indeed, the $\varepsilon$-cone property is equivalent for a set to have a boundary which is uniformly Lipschitz. That means that, locally, $\partial \omega$ can be written as a graph of a Lipschitzian function, the Lipschitz constant $L$ being the same for all boundary points. More precisely, we will use later on the following property:

$\forall X \in \partial \omega$, there exist reference axis centered at $X$ with a third (vertical) vector $\xi_{X}$ and a function $\psi$ Lipschitzian, with a Lipschitz constant $L=\cot \varepsilon / 2$ such that, if we denote by $C_{\varepsilon}$ the cylinder which have for base the disk $D_{\varepsilon}$ of radius $\varepsilon \tan \varepsilon$ and for height $[-\varepsilon, \varepsilon]$, we have

$$
\left\{\begin{array}{l}
\partial \omega \cap C_{\varepsilon}=\left\{(x, y, z) \in C_{\varepsilon} / z=\psi(x, y) \quad(x, y) \in D_{\varepsilon}\right\} \\
\omega \cap C_{\varepsilon}=\left\{(x, y, z) \in C_{\varepsilon} / z>\psi(x, y) \quad(x, y) \in D_{\varepsilon}\right\}
\end{array}\right.
$$

This property is well known, one can find a proof e.g. in [4] or [8].

Let us now precise the class of admissible sets for our maximization problem. We include in this definition the two constraints presented above:

Definition 1.2. Let us consider a fixed positive number $\varepsilon$, we will denote by $\mathcal{C}=\mathcal{C}\left(\varepsilon, V_{0}, z_{0}\right)$ the class of admissible sets defined by:

$$
\mathcal{C}=\left\{\omega \subset \mathbb{R}^{3}, \omega \text { has the } \varepsilon \text {-cone property },|\omega| \leq V_{0}, \omega \text { is located above the plane } z=z_{0}\right\} .
$$

Since the linear form in the right-hand side of (7) is calculated using a boundary integral over $\partial \omega$, we will need the uniform boundedness of the area for the sets in the class $\mathcal{C}$ :

Proposition 1.3. There exists a constant $C$ depending only on $\varepsilon$ and $V_{0}$ such that

$$
\forall \omega \in \mathcal{C} \quad|\partial \omega| \leq C .
$$

Proof. Let us introduce $\tilde{\varepsilon}=\min (\varepsilon, \varepsilon \tan \varepsilon)$. Since $\omega$ has the $\varepsilon$-cone property, it has also the $\tilde{\varepsilon}$-cone property.

Let us recover $\mathbb{R}^{3}$ with cubes (with disjoints interiors) of side length $\frac{\tilde{\varepsilon}}{\sqrt{3}}$. We denote by $\mathcal{N}$ the collection of those cubes which meet $\partial \omega$ and $N$ the cardinal of $\mathcal{N}$ ( $N$ could be a priori infinite, but the proof will show that it is uniformly bounded for all sets in the class $\mathcal{C}$ ).

Let $K_{1}$ be a cube in $\mathcal{N}, X_{1} \in K_{1} \cap \partial \omega$. and $C_{1}$ the cone $C\left(X_{1}, \xi_{X_{1}}, \tilde{\varepsilon}\right)$ which is contained in $\omega$ by $\tilde{\varepsilon}$-cone property. Since the diameter of this cone is $\tilde{\varepsilon}$ and the diameter of an elementary cube is also $\tilde{\varepsilon}$, by construction $C_{1}$ can meet at most 27 cubes of the covering. Now the alternative is the following:

- either $\omega$ is contained in the union of these 27 cones and we stop,

- or it is not the case and we do again the same process by choosing a new cube $K_{2}$, not in the previous list, a new point $X_{2}$ and a new cone $C_{2}$ disjoint from $C_{1}$.

Since the volume $V_{0}$ of $\omega$ is greater or equal to the sum of the (fixed) volume of each cones, this process has to stop. This shows that the number $N$ of cubes which meet $\omega$ is bounded by $N \leq \frac{V_{0}}{27 \mid C_{\varepsilon}}$ where $\left|C_{\varepsilon}\right|$ is the volume of a cone.

Now, since

$$
|\partial \omega|=\sum_{K \in \mathcal{N}}|\partial \omega \cap K| \leq N \max _{K \in \mathcal{N}}|\partial \omega \cap K|,
$$

it is enough to estimate the area of $\partial \omega$ on each elementary cube. But, if $X \in K \cap \partial \omega$, we have, according to the respective dimensions

$$
\partial \omega \cap K \subset \partial \omega \cap B(X, \tilde{\varepsilon}) .
$$


So, using (15), we have

$$
|\partial \omega \cap B(X, \tilde{\varepsilon})|=\iint_{D_{\varepsilon}} \sqrt{1+\left(\frac{\partial \psi}{\partial x}\right)^{2}+\left(\frac{\partial \psi}{\partial y}\right)^{2}} \mathrm{~d} x \mathrm{~d} y \leq \sqrt{1+2 L^{2}} \pi \tilde{\varepsilon}^{2}
$$

what implies the desired result.

\section{Remark 1.4.}

1. Because of the volume constraint, it is easy to verify that the number of cubes which recover a set $\omega$ in $\mathcal{C}$ is also uniformly bounded by a constant $N_{1}$ depending only on $\varepsilon$ and $V_{0}$.

2. The diameter of sets in the class $\mathcal{C}$ is not uniformly bounded: we can imagine two balls of fixed radius going away each other. On the other hand, it is easy to prove, using the covering, that the diameter of each connected component of $\omega$ must be uniformly bounded.

3. Because of the $\varepsilon$-cone property, the volume of each connected component of $\omega$ is bounded from below (by the volume of an elementary cone), therefore the number of connected component of $\omega$ is uniformly bounded by an integer that we will denote by $N_{0}$.

\subsection{Estimation of the functional $J$}

Since we are interested in the maximization of the functional $J$, we will first prove that it is estimated from above:

Proposition 1.5. There exists a constant $C^{\prime}$, depending only on the data $\varepsilon, V_{0}, z_{0}, M_{0}$ such that

$$
\forall \omega \in \mathcal{C} \quad J(\omega) \leq C^{\prime} .
$$

Proof. Let $\omega$ be a fixed set in the class $\mathcal{C}$, we denote by $\Gamma_{0}$ its boundary and $\Omega$ the complementary of the wheel ( $\Gamma$ being the boundary of $\Omega$ ). We use here the notations of section 1 (see (9)). By maximum principle and (8), we have

$$
\sup _{\Omega}|v|=\sup _{\Gamma}\left|u_{\infty}\right| \leq \frac{1}{4 \pi} \frac{\left|M_{0}\right||\partial \omega|}{d\left(\Gamma_{0}, \Gamma\right)}
$$

where $d\left(\Gamma_{0}, \Gamma\right):=\inf \left\{\left|X_{0}-X_{1}\right|, X_{0} \in \Gamma_{0}, X_{1} \in \Gamma\right\}$ denotes the distance between $\Gamma_{0}$ and $\Gamma$ which is bounded from below by some positive distance $\delta_{1}$.

Now, let us fix an open neighbourhood $\hat{S}$ of $S$ which does not intersect $\Omega_{0}$ and $K$. In the same way, we have

$$
\sup _{\hat{S}}\left|u_{\infty}\right| \leq \frac{1}{4 \pi} \frac{\left|M_{0}\right||\partial \omega|}{d\left(\Gamma_{0}, \hat{S}\right)}
$$

with $d\left(\Gamma_{0}, \hat{S}\right)$ bounded from below by some positive distance $\delta_{2}$.

This yields the following estimate for $\varphi$ on $\hat{S}$ :

$$
\forall X \in \hat{S} \quad|\varphi(X)| \leq \frac{\left|M_{0}\right||\partial \omega|}{4 \pi}\left(\frac{1}{d\left(\Gamma_{0}, \Gamma\right)}+\frac{1}{d\left(\Gamma_{0}, \hat{S}\right)}\right) .
$$

In (17), the right-hand side can be bounded independently of $\omega$, thanks to Proposition 1.3 and the previous remarks.

We deduce now from (17) and from usual representation formulae for harmonic functions, an uniform estimate for $|\nabla \varphi|^{2}$ on $S$, and Proposition 1.5 follows. 


\subsection{Continuity with respect to the domain}

As it is usual in many shape optimization problems, we choose to work with the Hausdorff distance of open sets. Let us recall its definition:

Definition 1.6. Let $K_{1}$ and $K_{2}$ be compact subsets in $\mathbb{R}^{3}$, we define their Hausdorff distance by

$$
d_{H}\left(K_{1}, K_{2}\right):=\sup _{X \in \mathbb{R}^{3}}\left|d\left(X, K_{1}\right)-d\left(X, K_{2}\right)\right|
$$

If $\omega_{1}$ and $\omega_{2}$ are two bounded open sets in $\mathbb{R}^{3}$, we fix a ball $B$ containing both and we set

$$
d_{H}\left(\omega_{1}, \omega_{2}\right):=d_{H}\left(B \backslash \omega_{1}, B \backslash \omega_{2}\right) .
$$

(it is easy to verify that this definition doesnot depend on the choice of the ball $B$ containing the two open sets).

We will say that a sequence $\omega_{n}$ Hausdorff-converges to $\omega$ if $d_{H}\left(\omega_{n}, \omega\right) \rightarrow 0$.

Let us recall some classical properties of the Hausdorff convergence, for the proofs we refer to [8, 11], or [3].

\section{Proposition 1.7.}

(i) (compactness) Let $B$ be a fixed ball and $\omega_{n}$ a sequence of open sets contained in $B$. Then, there exists a subsequence $\omega_{n_{k}}$ and an open set $\omega \subset B$ such that $\omega_{n_{k}}$ Hausdorff-converges to $\omega$.

(ii) Let $\omega_{n}$ be a sequence of open sets which Hausdorff-converges to $\omega$ and $X \in \partial \omega$. Then, there exists a sequence of points $X_{n} \in \partial \omega_{n}$ which converges to $X$.

(iii) Let $\omega_{n}$ be a sequence of open sets which have the $\varepsilon$-cone property (for some fixed $\varepsilon>0$ ). We assume that $\omega_{n}$ Hausdorff-converges to $\omega$. Then, $\omega$ has the $\varepsilon$-cone property. Moreover, $\chi_{\omega_{n}}$ (the characteristic function of $\left.\omega_{n}\right)$ converges in $L_{l o c}^{1}\left(\mathbb{R}^{3}\right)$ to $\chi_{\omega}$.

The property (iii) shows, in particular, that the class $\mathcal{C}$ defined above (see Def. 1.2) is closed for the Hausdorffconvergence since the two constraints will also pass to the limit:

- $\left|\omega_{n}\right|=\int_{B} \chi_{\omega_{n}} \rightarrow \int_{B} \chi_{\omega}=|\omega|$

- $\omega_{n} \subset\left\{z \geq z_{0}\right\} \quad \Longrightarrow \omega \subset\left\{z \geq z_{0}\right\}$ (since Hausdorff convergence preserves the inclusion).

For every open set $\omega$ in the class $\mathcal{C}$, we will denote by $L_{\omega}$ the linear form defined on $W_{0}^{1}(\Omega)$ by $(6)$. We will also denote by $\varphi_{\omega}$ the solution of $(7)$, with right-hand side $L_{\omega}$. In the following result, we consider two kinds of situations: a sequence of domains $\omega_{n}$ which goes to infinity and a sequence of domains which Hausdorffconverges.

\section{Theorem 1.8.}

1. If $\omega_{n}$ is a sequence of sets in the class $\mathcal{C}$ which goes to infinity (in the sense that $\left.d\left(0, \omega_{n}\right) \rightarrow+\infty\right)$, then $\varphi_{\omega_{n}}$ converges uniformly to 0 , as all its derivatives, on $S$.

2. If $\omega_{n}$ is a sequence of sets in the class $\mathcal{C}$ which Hausdorff-converges to $\omega$, then $\varphi_{\omega_{n}}$ converges uniformly to $\varphi_{\omega}$, and all the derivatives of $\varphi_{\omega_{n}}$ converges uniformly to the corresponding derivatives of $\varphi_{\omega}$, on $S$.

Proof. The first case follows immediately from formula (17). Indeed, if $\omega_{n}$ goes to infinity, it is the same for each distance $d\left(\Gamma_{0, n}, \Gamma\right)$ and $d\left(\Gamma_{0, n}, \hat{S}\right)$ and the result follows for $\varphi_{\omega_{n}}$ according to (17) since $\left|\partial \omega_{n}\right|$ is uniformly bounded. Now, the result is also true for the derivatives due to classical properties of sequences of harmonic functions (Harnack's inequalities).

The second case is a little bit more complicated. We write, as in $(9), \varphi_{\omega_{n}}=u_{\infty}^{\omega_{n}}-v^{\omega_{n}}$ where

$$
u_{\infty}^{\omega_{n}}(X)=\frac{1}{4 \pi} \int_{\partial \omega_{n}} \frac{\mathbf{M}_{\mathbf{0}} \cdot \mathbf{n}}{|X-Y|} \mathrm{d} \sigma_{Y}
$$


and $v^{\omega_{n}}$ is the harmonic function, taking the value $u_{\infty}^{\omega_{n}}$ on $\Gamma$ and vanishing at infinity (and similarly replacing $\omega_{n}$ by $\left.\omega\right)$.

Let us first prove that $u_{\infty}^{\omega_{n}}$ converge uniformly to $u_{\infty}^{\omega}$ on every compact subset $K$ of $(\bar{\omega})^{c}$ (according to classical properties of Hausdorff convergence, such a compact is included in $\left(\overline{\omega_{n}}\right)^{c}$ for $n$ large enough). Let $\hat{X}$ be fixed on $K$, we denote by $w(Y)$ the function $\frac{1}{|\hat{X}-Y|}$. We begin to work locally. Let $X \in \partial \omega$ and $\xi_{X}$ the direction of the cone associated to $X$. According to Proposition 1.7, there exists a sequence of points $X_{n}$, each lying on the boundary of $\omega_{n}$, which converge to $X$. Moreover, we know ( $c f$. [8] or [3]) that we are always able to choose the sequence of associated cone directions $\xi_{X_{n}}$ in order that they converge to $\xi_{X}$. Therefore, for $n$ large enough, the boundary of $\omega_{n}$ in a neighbourhood of $X_{n}$ can be written as a graph of a function $\psi_{n}$ $(L+1)$-Lipschitzian in some local coordinate system $\mathcal{R}_{X}$ associated to the point $X$ with vertical vector $\xi_{X}$ (see (15)). The functions $\psi_{n}$ being uniformly Lipschitzian, by Ascoli's Theorem we can extract a subsequence, still denoted by $\psi_{n}$, which converges uniformly to a Lipschitzian function $\psi$. It is classical then that $\psi$ defines locally the boundary of $\omega$ in a neighbourhood of $X$. Since $\psi$ is the only accumulation point of the sequence $\psi_{n}$, the whole sequence converges to $\psi$.

Let us denote by $\gamma_{n}$ (resp. $\gamma$ ), the intersection of $\partial \omega_{n}$ (resp. $\partial \omega$ ) with the cylinder $C_{\varepsilon}$, neighbourhood of $X$ in which the parametrizations defined by $\psi_{n}$ and $\psi$ are valid. Denoting by $M_{0}=(A, B, C)^{T}$, we have in $\mathcal{R}_{X}$ :

$$
\int_{\gamma_{n}} w(\sigma) M_{0} . n(\sigma) \mathrm{d} \sigma=\int_{D_{\varepsilon}} w\left(x, y, \psi_{n}(x, y)\right)\left(-A \frac{\partial \psi_{n}}{\partial x}-B \frac{\partial \psi_{n}}{\partial y}+C\right) \mathrm{d} x \mathrm{~d} y
$$

and similarly for $\int_{\gamma} w(\sigma) M_{0} . n(\sigma) \mathrm{d} \sigma$.

On one hand, $w\left(x, y, \psi_{n}(x, y)\right)$ converge uniformly to $w(x, y, \psi(x, y))$ on $D_{\varepsilon}$ according to the beginning of the proof. On the other hand, $\psi_{n}$ converges uniformly to $\psi, \frac{\partial \psi_{n}}{\partial x}$ and $\frac{\partial \psi_{n}}{\partial y}$ converge in the sense of distributions to $\frac{\partial \psi}{\partial x}$ and $\frac{\partial \psi}{\partial y}$ respectively. But, since $\frac{\partial \psi_{n}}{\partial x}$ and $\frac{\partial \psi_{n}}{\partial y}$ are bounded in $L^{\infty}$ (because of the uniform Lipschitz property of the functions $\psi_{n}$ ), the convergence takes place indeed in $L^{\infty}$ weak-*. Therefore the duality pair $\left\langle w\left(x, y, \psi_{n}(x, y)\right),-A \frac{\partial \psi_{n}}{\partial x}-B \frac{\partial \psi_{n}}{\partial y}+C\right\rangle$ converge to $\left\langle w(x, y, \psi(x, y)),-A \frac{\partial \psi}{\partial x}-B \frac{\partial \psi}{\partial y}+C\right\rangle$ what proves the result locally. We conclude thanks to a finite covering of $\partial \omega$ by neighbourhoods of the kind $C_{\varepsilon}$ and by using a corresponding partition of the unity. We have proved indeed that $u_{\infty}^{\omega_{n}}$ simply converge to $u_{\infty}^{\omega}$, but since it is a bounded sequence of harmonic functions, we have in fact uniform convergence on every compact.

Now, by maximum principle, the convergence of $u_{\infty}^{\omega_{n}}$ to $u_{\infty}^{\omega}$ implies convergence of $v^{\omega_{n}}$ to $v^{\omega}$ on every compact subset of $(\bar{\omega})^{c}$.

So, $\varphi_{\omega_{n}}$ converge to $\varphi_{\omega}$ on every compact subset of $(\bar{\omega})^{c}$. By Harnack inequalities, the convergence takes place also for the derivatives of $\varphi_{\omega_{n}}$.

\subsection{The existence result}

We can now claim the main result of this section:

Theorem 1.9. The functional $J$ defined in (12) achieves its maximum on the class $\mathcal{C}$.

Proof. Let us consider a maximizing sequence $\omega_{n}$. According to the Remark 1.4 above, each open set $\omega_{n}$ has at most $N_{0}$ connected components. We can thus decompose it as

$$
\omega_{n}=\omega_{n}^{1} \cup \omega_{n}^{2} \cup \ldots \cup \omega_{n}^{N_{0}}
$$

where in the decomposition (18), some connected components could be empty and all the connected components are disjoints. By the linearity of the problem, we can write in a similar way

$$
L_{\omega_{n}}=L_{\omega_{n}^{1}}+L_{\omega_{n}^{2}}+\ldots+L_{\omega_{n}^{N_{0}}}
$$


where, by convention $L_{\emptyset}=0$. In the previous decomposition, we use the fact that the open set $\omega$ being Lipschitzian, two different connected components cannot have a common boundary part $\left(\omega_{n}\right.$ has to be on one side of its boundary). Still by a linearity argument, we deduce from (19), the corresponding decomposition for $\varphi_{\omega_{n}}$ :

$$
\varphi_{\omega_{n}}=\varphi_{\omega_{n}^{1}}+\varphi_{\omega_{n}^{2}}+\ldots+\varphi_{\omega_{n}^{N_{0}}}
$$

(with $\varphi_{\emptyset}=0$ ). We are now working with each connected component separately. Let us study the sequence $\omega_{n}^{1}$. There are two possibilities:

- either, there exists a subsequence $\omega_{n_{k}}^{1}$ which stay confined in a ball. In this case, we can extract a subsequence which converges in the Hausdorff sense to an open set $\omega^{1}$ (which has the $\varepsilon$-cone property).

- or the whole sequence $\omega_{n}^{1}$ goes to infinity, and we set $\omega^{1}=\emptyset$.

By doing the same for each connected component, we are able, after a finite number of such operations, to construct a subsequence $\omega_{n_{k}}$ of the maximizing sequence $\omega_{n}$ and an open set $\omega \in \mathcal{C}$ defined by

$$
\omega=\omega^{1} \cup \omega^{2} \cup \ldots \cup \omega^{N_{0}}
$$

According to Theorem 1.8 and (20) we have that $\varphi_{\omega_{n_{k}}}$ converge in the harmonic sense to $\varphi_{\omega}$. Therefore $J\left(\omega_{n_{k}}\right)$ converge to $J(\omega)$ which yields the desired result.

Remark 1.10. It is clear that the empty set could not be a maximiser of $J$. Indeed, we are always able to exhibit an open set $\omega$ in the class $\mathcal{C}$ such that $J(\omega)>0=J(\emptyset)$ (see Sect. 3). Therefore, the above existence result yields a global maximum in the class $\mathcal{C}$. Nevertheless, since our numerical algorithm, described in the next section, does not change the topology of the initial guess, we cannot see if pieces of a minimizing sequence would like to go to infinity. Moreover, it could also converge to a local minimizer, see also final comments.

\section{Discretization of the Problem}

\subsection{Introduction}

In this section, we want to explain the way we deform the successive magnets in order to increase the value of the functional $J$. We follow the same idea as in $[9,10]$. The method consists in constructing a sequence $\left(\Gamma_{0}^{k}, \varphi^{k}, V^{k}\right)_{k=1, \ldots, n}$ where

- $\Gamma_{0}^{k}$ is a triangular mesh representing the magnet at the $k$-th iteration. Each triangle $T_{l}$ is parametrized by a reference triangle $\hat{T}$ of coordinates $\xi$ and $\eta$. Therefore, if $X^{l, 1}, X^{l, 2}, X^{l, 3}$ are the vertices of the triangle $T^{l}$, each point of the triangle can be represented by

$$
X=X(\xi, \eta)=\sum_{i=1}^{3} X^{l, i} N_{i}(\xi, \eta)
$$

where

$$
N_{1}(\xi, \eta)=1-\xi-\eta, \quad N_{2}(\xi, \eta)=\xi, \quad N_{3}(\xi, \eta)=\eta
$$

In the sequel, we will denote by $X_{\xi}$ and $X_{\eta}$ the derivative of $X$ with respect to $\xi$ and $\eta$, that is to say, with the previous formulae:

$$
X_{\xi}=X^{l, 2}-X^{l, 1} \quad X_{\eta}=X^{l, 3}-X^{l, 1}
$$

- $\varphi_{k}^{T}$ and $\varphi_{k}^{H}$ denote the magnetic scalar potentials (in each situation) created by the magnet whose boundary is $\Gamma_{0}^{k}$. Each are solutions of a problem like (7). 
- $V^{k}$ denotes the vector field which gives the direction of the displacement at each node of $\Gamma_{0}^{k}$. More precisely, if the nodes are denoted by $\left(X_{i}^{k}\right)_{i=1 . . M}$, for each node $X_{i}^{k}$, we choose the direction $V_{i}^{k}$ as the weighted average of the exterior normal vectors to triangles having $X_{i}^{k}$ as a vertex.

Now, we have to decide how much we will deform the magnet $\Gamma_{0}^{k}$ at each vertices in the direction $V_{i}^{k}$. So, we introduce the unknowns $\left(u_{i}\right)_{i=1 . . M}$ which are the intensity of the deformation of the nodes $X_{i}^{k}$ in the direction $V_{i}^{k}$. In other terms, we have to find a vector in $\mathbb{R}^{M}: U=\left(u_{i}\right)_{i=1 . . M}$ which maximizes (at least locally) the functional $J\left(\Gamma_{0}^{U}\right):=J^{k}(U)$ where $\Gamma_{0}^{U}$ is the boundary of the magnet whose nodes are the points $X_{i}^{U}:=X_{i}^{k}+u_{i} V_{i}^{k}$.

We simply improve the functional $J^{k}(U)$ with the help of a quasi-Newton B.F.G.S. method (see e.g. [2] or [6]). At each iteration we must compute the gradient with respect to $U$ of the functional $J^{k}$ at $U=0$.

\subsection{Computation of the discretized gradient}

We give first the value of the gradient for the continuous problem. It helps to well understand the formula for the discretized problem. We use here the classical tool of the derivative with respect to the domain. See references below.

Proposition 2.1. Let $\varphi$ the solution of (7), and $j$ the functional defined by

$$
j\left(\Omega_{0}\right)=\int_{S}|\nabla \varphi(X)|^{2} \mathrm{~d} X .
$$

Assume that $\Omega_{0}$ is of class $C^{2}$, then the derivative of $j$ at $\Omega_{0}$ with respect to a deformation of the boundary induced by the vector field $V$ is given by

$$
d j\left(\Omega_{0}, V\right)=-\int_{\Gamma_{0}} M_{0} \cdot \nabla_{\Gamma_{0}}(V \cdot \mathbf{n}) p \mathrm{~d} \sigma+\int_{\Gamma_{0}} M_{0} \cdot \mathbf{n} p H(V \cdot \mathbf{n}) \mathrm{d} \sigma+\int_{\Gamma_{0}} \frac{\partial p}{\partial n} M_{0} \cdot \mathbf{n}(V \cdot \mathbf{n}) \mathrm{d} \sigma
$$

where $\nabla_{\Gamma_{0}}$ denotes the tangential gradient on the boundary $\Gamma_{0}, H$ is the mean curvature of $\Gamma_{0}$ and $p$ is the adjoint state, solution of the following problem:

$$
\left\{\begin{aligned}
-\Delta p & =L_{S} & & \text { in } \Omega \\
p & =0 & & \text { on } \partial \Omega \\
p(X) & \rightarrow & & \text { when }|X| \rightarrow+\infty
\end{aligned}\right.
$$

where $L_{S}$ is the linear form defined on $W_{0}^{1}(\Omega)$ by

$$
\left\langle L_{S}, w\right\rangle:=\left\langle-\nabla \chi_{S} \cdot \nabla \varphi, w\right\rangle=\sum_{i=1}^{3} \int_{S} \frac{\partial}{\partial x_{i}}\left(\frac{\partial \varphi}{\partial x_{i}} w\right) \mathrm{d} \sigma_{S} .
$$

The proof follows straigthforward calculations and classical formulae given in the papers of Simon $[12,13]$ or the books of Sokolowski-Zolesio [14] or Henrot-Pierre [8]. We omit it here.

We can easily deduce, from the previous formula (22), the expression of the derivative, with respect to the domain, of the functional $J$ defined in (12). We can imagine that this formula is not very suitable from a computational point of view, due to terms involving the mean curvature or the tangential gradient. That is the reason why we have chosen to compute directly the gradient of the discretized functional rather than discretizing the continuous gradient.

We want to compute the gradient of the functional $j$ defined in (21) with respect to the variable $u_{i}$ at $u=0$. It means that we deform the initial magnet by moving only the $i$ th node of some distance $h_{i}$, we set $\Omega_{0}^{h_{i}}$ the new magnet, and we have to calculate

$$
\lim _{h_{i} \rightarrow 0} \frac{j\left(\Omega_{0}^{h_{i}}\right)-j\left(\Omega_{0}\right)}{h_{i}} .
$$


The computation involves the function $\varphi_{i}$ solution of (7) with a right-hand side $L_{0}^{i}$ defined by (6) on the magnet $\Omega_{0}^{h_{i}}$. First, we want to compute the limit, when $h_{i} \rightarrow 0$ of the function $w^{h_{i}}:=\frac{\varphi_{i}-\varphi}{h_{i}}$. Let us decompose this quotient as (see (9))

with

$$
\frac{\varphi_{i}-\varphi}{h_{i}}=\frac{u_{\infty}^{i}-u_{\infty}}{h_{i}}+\frac{v_{i}-v}{h_{i}}
$$

$$
u_{\infty}^{i}(X)=\frac{1}{4 \pi} \int_{\partial \Omega_{0}^{h_{i}}} \frac{\mathbf{M}_{\mathbf{0}} \cdot \mathbf{n}}{|X-Y|} \mathrm{d} \sigma_{Y}
$$

and $v_{i}$ is the harmonic function, taking the value $u_{\infty}^{i}$ on $\Gamma$ and vanishing at infinity (and the same for $\Omega_{0}$ ).

Lemma 2.2. The sequence of functions $\frac{u_{\infty}^{i}-u_{\infty}}{h_{i}}$ converge (uniformly on every compact subset of the exterior of $\left.\Omega_{0}\right)$, when $h_{i} \rightarrow 0$, to the function $U_{i}$ defined by

$$
U_{i}(X)=\left\langle L_{i}^{\prime}, \frac{1}{|X-Y|}\right\rangle
$$

where $L_{i}^{\prime}$ is the linear form defined by

$$
\begin{gathered}
\left\langle L_{i}^{\prime}, w\right\rangle:=\sum_{j} \int_{\hat{T}}\left\{\mathbf{M}_{\mathbf{0}} \cdot \mathbf{Q}_{\mathbf{j}} w+(1-\xi-\eta) \mathbf{M}_{\mathbf{0}} \cdot \mathbf{n}_{\mathbf{j}} \nabla w \cdot V_{i}\right\}\left|X_{\xi} \wedge X_{\eta}\right| \mathrm{d} \xi \mathrm{d} \eta \\
+\sum_{j} \int_{\hat{T}}\left\{\mathbf{M}_{\mathbf{0}} \cdot \mathbf{n}_{\mathbf{j}}\right\} w \mathbf{n}_{\mathbf{j}} \cdot\left(V_{i} \wedge\left(X^{j_{1}}-X^{j_{2}}\right)\right) \mathrm{d} \xi \mathrm{d} \eta
\end{gathered}
$$

where $\hat{T}$ is the reference triangle and $\mathbf{Q}_{\mathbf{j}}$ is the vector defined by

$$
\mathbf{Q}_{\mathbf{j}}=\frac{2\left(V_{i} \cdot \mathbf{n}_{\mathbf{j}}\right)\left(\mathbf{n}_{\mathbf{j}} \wedge\left(X^{j_{1}}-X^{j_{2}}\right)\right.}{\left|T_{j}\right|}
$$

the above sum is done on all the triangles $T_{j}$ containing $X^{i}$ as a vertex, $X^{j_{1}}$ and $X^{j_{2}}$ being the two other vertices of $T_{j}, \mathbf{n}_{\mathbf{j}}$ its normal vector and $\left|T_{j}\right|$ the area of $T_{j}$. The vector $V_{i}$ is defined in Section 2.1; it is the weighted average of the normal vectors $\mathbf{n}_{\mathbf{j}}$.

Proof. Let $X$ be fixed in the proof and let us denote by $w(Y)$ the function $Y \mapsto \frac{1}{|X-Y|}$. By definition, we have

$$
R\left(h_{i}\right):=\frac{u_{\infty}^{i}(X)-u_{\infty}(X)}{h_{i}}=\frac{1}{h_{i}}\left[\int_{\Gamma_{0}^{i}} \mathbf{M}_{\mathbf{0}} \cdot \mathbf{n}_{\mathbf{h}_{\mathbf{i}}} w \mathrm{~d} \sigma_{i}-\int_{\Gamma_{0}} \mathbf{M}_{\mathbf{0}} \cdot \mathbf{n} w \mathrm{~d} \sigma\right]
$$

where $\Gamma_{0}^{i}$ is the boundary of the deformed magnet $\Omega_{0}^{h_{i}}$. It is clear on the previous formula (27) that the only contribution will come from the triangles which contain the $i$ th node $X^{i}$. So let us introduce $\Sigma T^{i}$ the set of triangles containing $X^{i}$ before the deformation and $\Sigma T^{h_{i}}$ the set of triangles containing $X^{i}$ after the deformation of intensity $h_{i}$. Therefore,

$$
R\left(h_{i}\right)=\frac{1}{h_{i}}\left[\int_{\Sigma T^{h_{i}}} \mathbf{M}_{\mathbf{0}} \cdot \mathbf{n}_{\mathbf{h}_{\mathbf{i}}} w \mathrm{~d} \sigma_{i}-\int_{\Sigma T^{i}} \mathbf{M}_{\mathbf{0}} \cdot \mathbf{n} w \mathrm{~d} \sigma\right]
$$

Let us consider one triangle, say $T_{j}$ in $\Sigma T^{i}$ whose vertices are $X^{i}, X^{j_{1}}, X^{j_{2}}$. The deformed triangle $T_{j}^{h_{i}}$ has for vertices $X^{i}+h_{i} V_{i}, X^{j_{1}}, X^{j_{2}}$. Coming back to the reference triangle yields:

$$
\int_{T_{j}^{h_{i}}} \mathbf{M}_{\mathbf{0}} \cdot \mathbf{n}_{\mathbf{j}, \mathbf{h}_{\mathbf{i}}} w \mathrm{~d} \sigma_{i}=\int_{\hat{T}} \mathbf{M}_{\mathbf{0}} \cdot \mathbf{n}_{\mathbf{j}, \mathbf{h}_{\mathbf{i}}} w\left[(1-\xi-\eta)\left(X^{i}+h_{i} V_{i}\right)+\xi X^{j_{1}}+\eta X^{j_{2}}\right]\left|X_{\xi}^{j, h_{i}} \wedge X_{\eta}^{j, h_{i}}\right| \mathrm{d} \xi \mathrm{d} \eta
$$


Now using the elementary expansions:

- $\mathbf{n}_{\mathbf{j}, \mathbf{h}_{\mathbf{i}}}=\mathbf{n}_{\mathbf{j}}+h_{i} \frac{\left(V_{i} \cdot \mathbf{n}_{\mathbf{j}}\right)\left(\mathbf{n}_{\mathbf{j}} \wedge\left(X^{j_{1}}-X^{j_{2}}\right)\right)}{2\left|T_{j}\right|}+o\left(h_{i}\right)$,

- $w\left[(1-\xi-\eta)\left(X^{i}+h_{i} V_{i}\right)+\xi X^{j_{1}}+\eta X^{j_{2}}\right]=w\left[(1-\xi-\eta) X^{i}+\xi X^{j_{1}}+\eta X^{j_{2}}\right]+(1-\xi-\eta) h_{i} \nabla w \cdot V_{i}+o\left(h_{i}\right)$,

- $\left|X_{\xi}^{j, h_{i}} \wedge X_{\eta}^{j, h_{i}}\right|=\left|\left(X^{j_{1}}-X^{i}-h_{i} V_{i}\right) \wedge\left(X^{j_{2}}-X^{i}-h_{i} V_{i}\right)\right|=\left|X_{\xi}^{j} \wedge X_{\eta}^{j}\right|+h_{i}\left(V_{i} \wedge\left(X^{j_{1}}-X^{j_{2}}\right)\right) . \mathbf{n}_{\mathbf{j}}+o\left(h_{i}\right)$

and adding on the different triangles containing $X^{i}$ gives the desired result thanks to straightforward calculations. Once again, we use the fact that, for a sequence of bounded harmonic functions, simple convergence implies uniform convergence on every compact sets.

Now, differentiation of the integral formula which defines $j$ gives:

Proposition 2.3. The derivative of the functional $j$ defined in (21) with respect to the variable $u_{i}$ at $u=0$ is given by

$$
\frac{\partial j}{\left.\partial u_{i}\right|_{u=0}}=2 \int_{S} \nabla \varphi \cdot \nabla w_{i} \mathrm{~d} X
$$

where $\varphi$ is the solution of (7) and $w_{i}$ is defined by

$$
w_{i}=U_{i}-\Phi_{i}
$$

where $U_{i}$ is defined in (25), (26) and $\Phi_{i}$ is the harmonic function taking the value $U_{i}$ on $\Gamma$ and vanishing at infinity.

\subsection{The adjoint problem}

As it is classical in optimal control problems, it is more convenient to express the previous derivative thanks to the use of an adjoint state. We will denote by $\chi_{S}$ the characteristic function of the regular set $S$. Therefore, $\nabla \chi_{S}$ is a (positive) measure supported by the boundary of $S$. In particular, we can consider the following linear form $L_{S}:=-\nabla \chi_{S} . \nabla \varphi$ which is well defined on the weighted Sobolev space $W_{0}^{1}(\Omega)$ by

$$
\left\langle L_{S}, w\right\rangle:=\left\langle-\nabla \chi_{S} \cdot \nabla \varphi, w\right\rangle=\sum_{i=1}^{3} \int_{S} \frac{\partial}{\partial x_{i}}\left(\frac{\partial \varphi}{\partial x_{i}} w\right) \mathrm{d} \sigma_{S}
$$

(notice that $\varphi$ is $C^{\infty}$ inside $\Omega$ so, in particular, on $S$ ). Let us now introduce the function $p$ solution of the following boundary value problem:

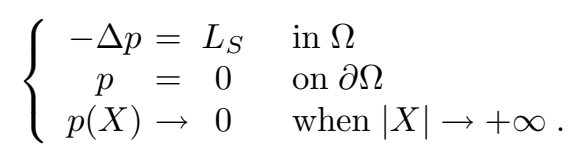

We can easily prove thanks to the Green formula (note that $p$ is $C^{\infty}$ on $\Omega_{0}$, so $\left\langle L_{i}^{\prime}, p\right\rangle$ is well defined):

Proposition 2.4. $\frac{\partial j}{\left.\partial u_{i}\right|_{u=0}}=\left\langle L_{i}^{\prime}, p\right\rangle$ with $L_{i}^{\prime}$ given in (26) and p solution of (32).

Of course, the interest of such a formula is to prevent computing each function $w_{i}$ : it is sufficient to solve only the direct and the adjoint problem to get the derivative of the functional into consideration. The expression of the whole derivative follows immediately from the previous formula by considering the two situations (faced with a tooth or a hollow) and adding the corresponding contributions. 


\subsection{Further comments}

- We decided here to compute ourselves the electromagnetic field in order to be able to use its gradient explicitely in the expression of the gradient of the functional. Of course, we have done a validation of our computations by comparison with commercial codes (Ampere and Ansys).

- As explained in the Introduction, we have chosen a boundary element method to solve problems (7) and (32). More precisely, we use a Galerkin method to solve the integral equations (11), by multiplying both sides by basis functions defined on the mesh and integrating over the boundary. The choice of this formulation is motivated by the fact that these problems are set in exterior domains. Moreover, it has another advantage. In the different steps of our algorithm, the magnet is modified, but this modification occurs only in the right-hand side of the linear equations we have to solve. More precisely, the matrix of the linear system obtained in the Galerkin procedure is the same, not only for the state and the adjoint equation at one step, but at every step. Therefore, we can write a LU decomposition of this matrix at the beginning of the procedure, each resolution of the state or the adjoint equation being very fast after.

- It is well known in boundary element methods that we have to compute, after discretization, some singular integrals due to the kernel $\frac{1}{X-Y}$ in the integral equation (11). We circumvent this difficulty by transforming these integrals, thanks to an integration by part, into boundary integrals (on the boundary of each triangle).

- The optimization algorithm that we used is the by-now classical Quasi-Newton BFGS methods (see e.g. [2, $6]$ ). We do the one-dimensional search with some steps of parabolic interpolation.

- The penalization parameter $r$ for the volume constraint is not fixed, but adaptative. It changes dynamically during the procedure.

- During the procedure, the mesh of the magnet is, of course, modified. So we have to create new triangles. If the area of a triangle becomes too large, we decide to split it into three new triangles using the center of mass as a new node. We write a limitation on the angles in order to avoid creation of thin triangles. It is much more complicated to remove one triangle in a mesh, so we do not try to remove too small triangles.

\section{NumericAl RESUltS}

We present the results for different initial geometries. First a table gives the values of the volume of the magnet and of the penalized functional after a certain number of iterations. The evolution of these quantities is also given in percentage. We have chosen to present the penalized functional, actually the penalization constant is adapted in such a way that the penalization term does not exceed $1 \%$ of the total functional. Then, we plot these relatives evolutions on a graphic. The solid line represents the penalized functional and the dashed line the volume. At last, we show three pictures of magnets.

\subsection{Parallelepiped}

\begin{tabular}{|c|c|c|c|c|}
\hline Iterations & Volume & \% Volume & Functional & \% Functional \\
\hline 1 & 7.99 & 0 & $6.78 \times 10^{7}$ & 0 \\
\hline 50 & 7.98 & -0.31 & $7.19 \times 10^{7}$ & 6.09 \\
\hline 100 & 8.00 & 0.03 & $7.44 \times 10^{7}$ & 9.77 \\
\hline 150 & 8.04 & 0.46 & $7.75 \times 10^{7}$ & 14.35 \\
\hline
\end{tabular}




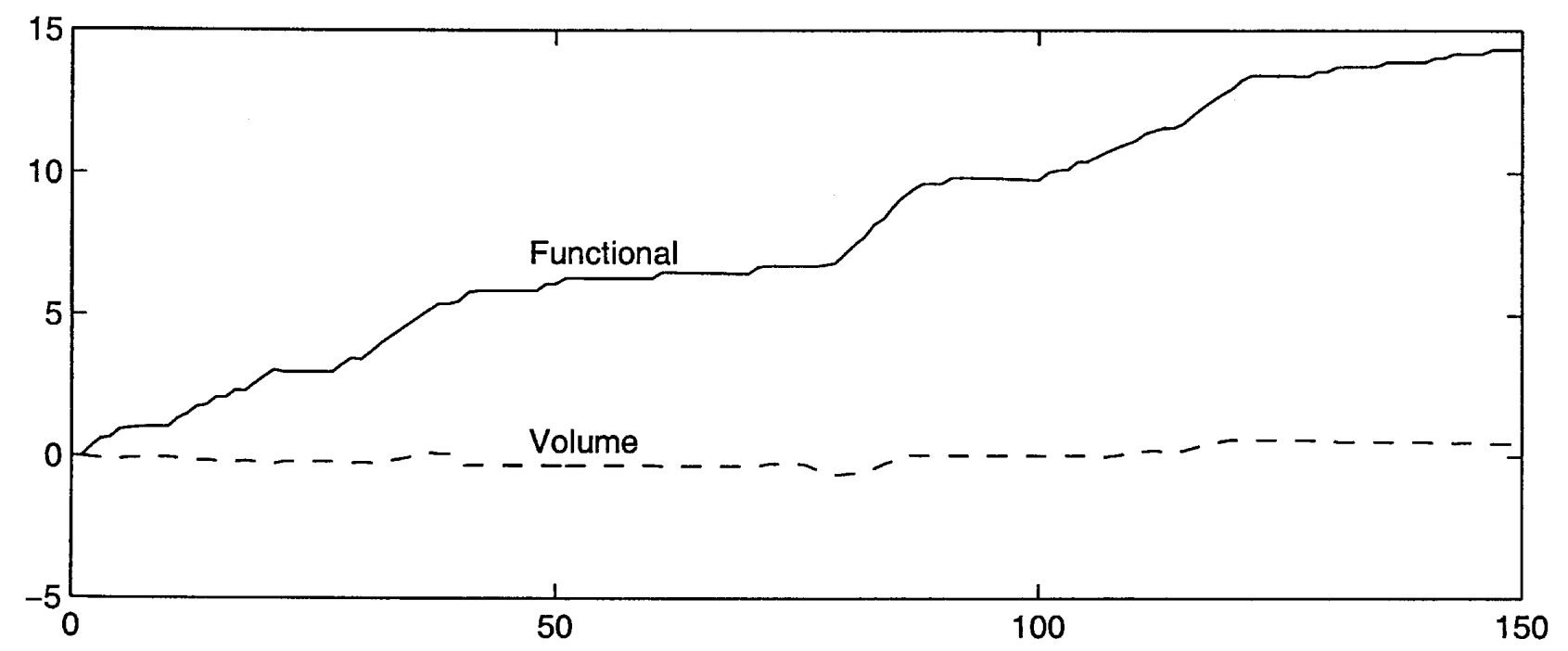

FigurE 2. Evolution of the functional and the volume in percentage.
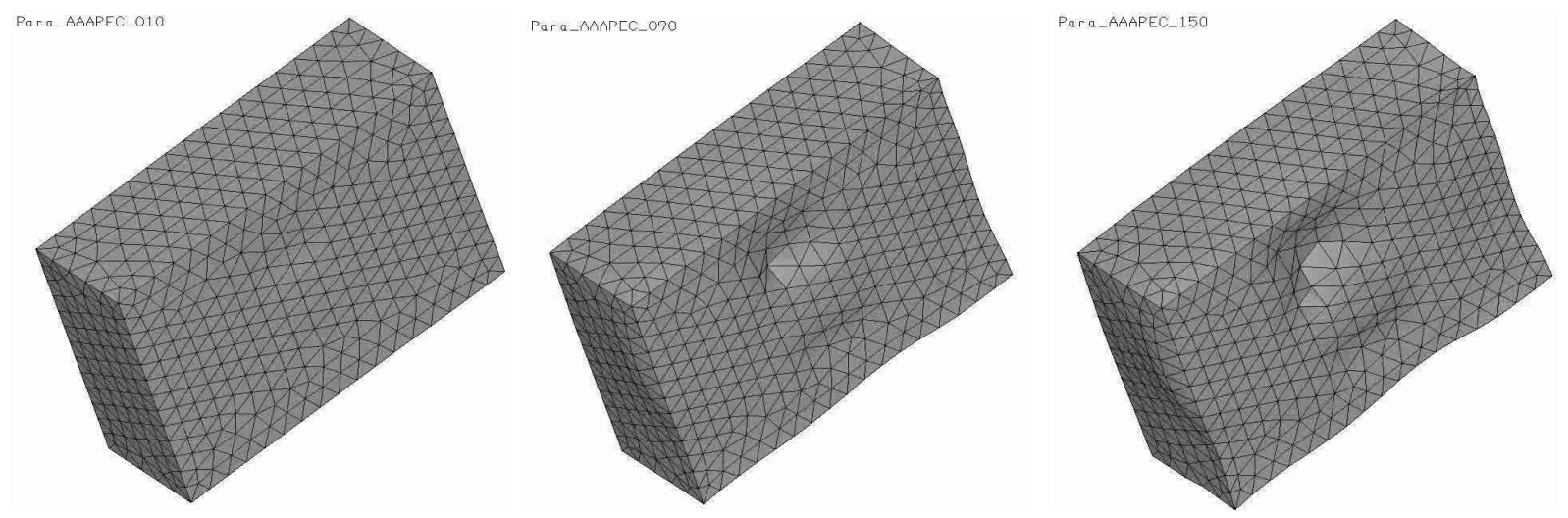

FigurE 3. Magnet after 10 iterations (left), 90 iterations (center), 150 iterations (right).

\subsection{Cylinder}

\begin{tabular}{|c|c|c|c|c|}
\hline Iterations & Volume & \% Volume & Functional & \% Functional \\
\hline 1 & 7.99 & 0 & $5.02 \times 10^{7}$ & 0 \\
\hline 50 & 7.95 & -0.51 & $5.32 \times 10^{7}$ & 5.99 \\
\hline 100 & 7.93 & -0.71 & $5.58 \times 10^{7}$ & 11.17 \\
\hline 150 & 7.95 & -0.54 & $5.83 \times 10^{7}$ & 16.20 \\
\hline
\end{tabular}




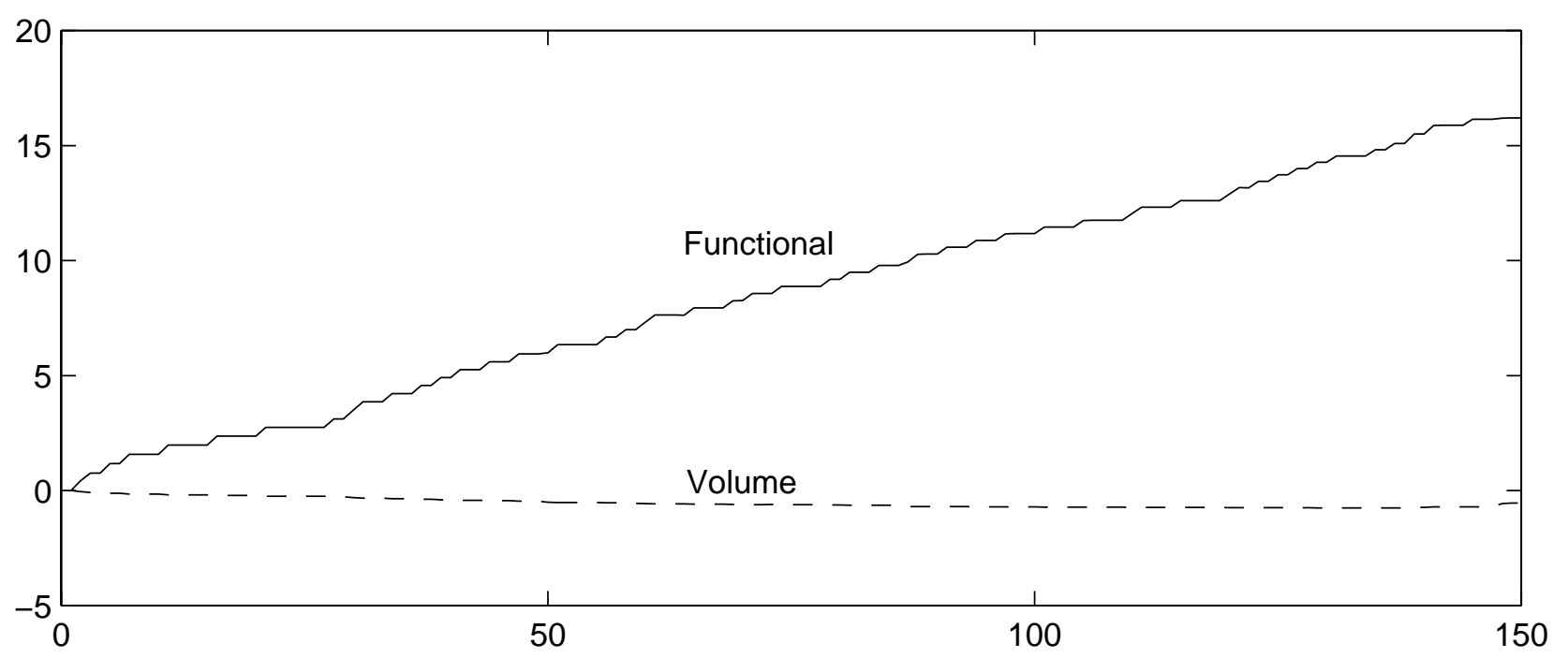

Figure 4. Evolution of the functional and the volume in percentage.
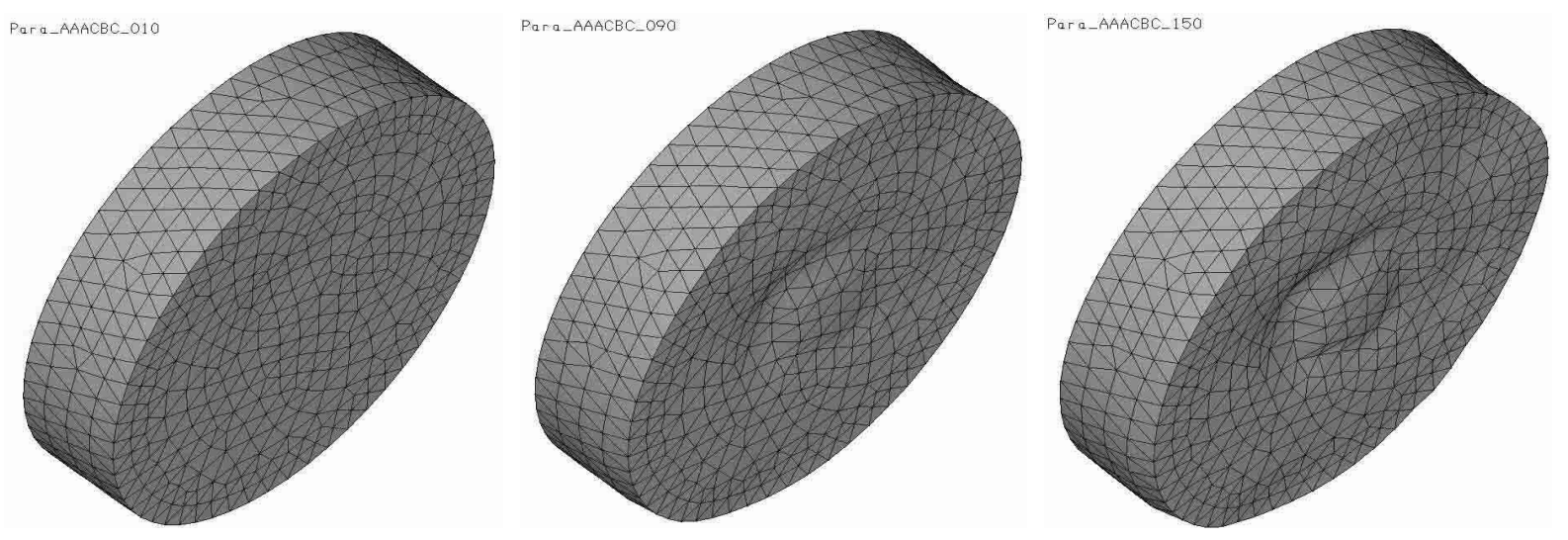

Figure 5. Magnet after 10 iterations (left), 90 iterations (center), 150 iterations (right).

\subsection{Bridge shape}

\begin{tabular}{|c|c|c|c|c|}
\hline Iterations & Volume & \% Volume & Functional & \% Functional \\
\hline 1 & 8.00 & 0 & $1.03 \times 10^{4}$ & 0 \\
\hline 10 & 7.91 & -1.05 & $1.77 \times 10^{4}$ & 72.2 \\
\hline 20 & 7.86 & -1.76 & $2.06 \times 10^{4}$ & 99.8 \\
\hline 25 & 7.83 & -2.11 & $2.25 \times 10^{4}$ & 118.9 \\
\hline
\end{tabular}




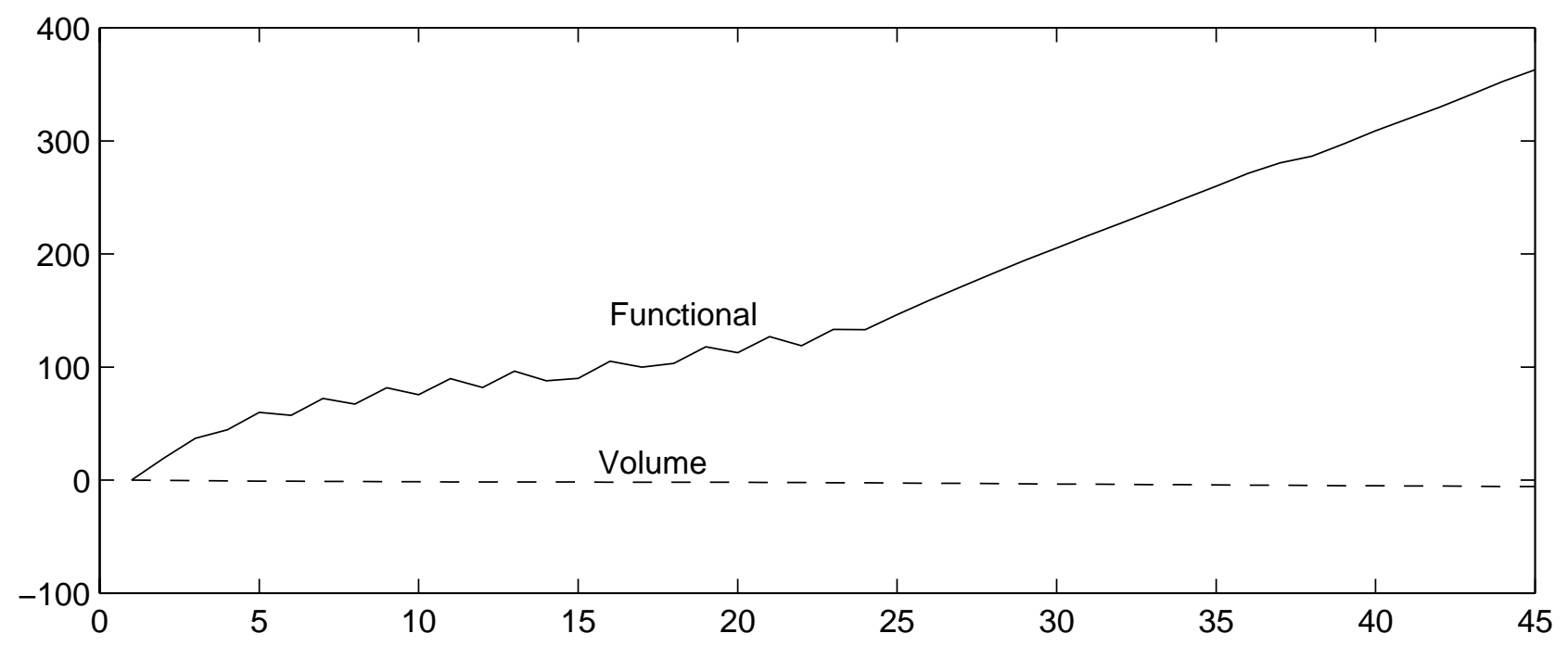

FiguRE 6. Evolution of the functional and the volume in percentage.
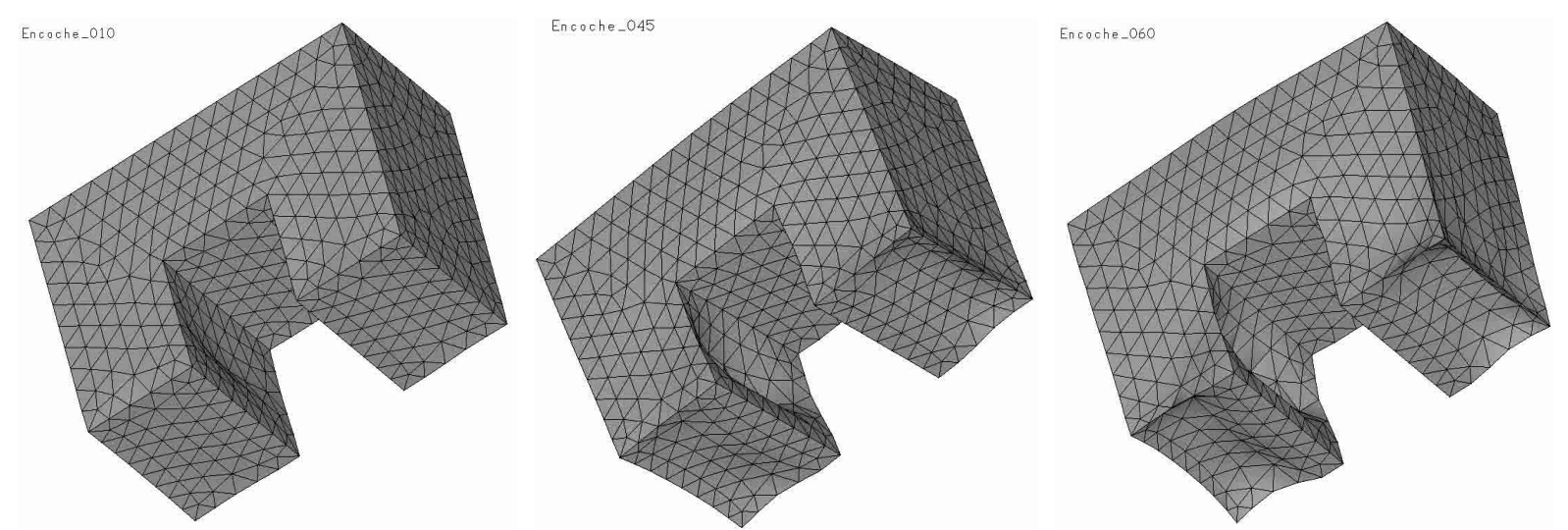

FiguRE 7. Magnet after 10 iterations (left), 45 iterations (center), 60 iterations (right).

\subsection{Final comments}

The above results show the efficiency of our method. Nevertheless, we were not able to observe convergence of the algorithm in any case. The main reason is that, after a certain number of steps, the mesh of the magnet is so deformed that some interpenetration or overlapping can occur. It is well visible in Figures 6 and 7 . For this last case, we can see that the results are no longer valids after iteration 25 due to problems in the magnet's mesh. We believe that the above pictures are still far from the optimum. If we really would try to improve our method to study the convergence of our algorithm, we would need a clever way to remesh the magnets. We did not do it by lack of time.

As we could guess, in each example, the top of the magnet is not changed. Modifications only occur on the bottom and the lateral faces. It also appears a kind of bulge. It looks as if the magnet would try to mimic the toothed wheel. 


\section{REFERENCES}

[1] S. Agmon, Lectures on Elliptic Boundary Value Problems. Van Nostrand Math Studies (1965).

[2] J. Baranger, Analyse Numérique. Hermann, Paris (1991).

[3] D. Chenais, On the existence of a solution in a domain identification problem. J. Math. Anal. Appl. 52 (1975) $189-289$.

[4] D. Chenais, Sur une famille de variétés à bord lipschitziennes, application à un problème d'identification de domaine. Ann. Inst. Fourier (Grenoble) 4 (1977) 201-231.

[5] R. Dautray and J.L. Lions (Eds.), Analyse mathématique et calcul numérique, Vol. I and II. Masson, Paris (1984).

[6] J.E. Dennis and R.B. Schnabel, Numerical Methods for unconstrained optimization. Prentice Hall (1983).

[7] E. Durand, Magnétostatique. Masson, Paris (1968).

[8] A. Henrot and M. Pierre, Optimisation de forme (to appear).

[9] M. Pierre and J.R. Roche, Computation of free sufaces in the electromagnetic shaping of liquid metals by optimization algorithms. Eur. J. Mech. B Fluids 10 (1991) 489-500.

[10] M. Pierre and J.R. Roche, Numerical simulation of tridimensional electromagnetic shaping of liquid metals. Numer. Math. 65 (1993) 203-217.

[11] O. Pironneau, Optimal shape design for elliptic systems. Springer Series in Computational Physics. Springer, New York (1984).

[12] J. Simon, Differentiation with respect to the domain in boundary value problems. Numer. Funct. Anal. Optim. 2 (1980) 649-687.

[13] J. Simon, Variations with respect to domain for Neumann condition. Proceedings of the 1986 IFAC Congress at Pasadena "Control of Distributed Parameter Systems".

[14] J. Sokolowski and J.P. Zolesio, Introduction to shape optimization: shape sensitity analysis. Springer Series in Computational Mathematics, Vol. 10, Springer, Berlin (1992).

To access this journal online:

www.edpsciences.org 